\title{
Ultrashort-pulse, terawatt, long-wave infrared lasers based on high-pressure $\mathrm{CO}_{2}$ amplifiers
}

\author{
Mikhail N. Polyanskiy*, Igor V. Pogorelsky, Marcus Babzien, Rotem Kupfer, and Mark A. Palmer \\ Accelerator Test Facility, Brookhaven National Laboratory, Upton, New York 11973, USA
}

\begin{abstract}
We discuss the state of the art, the ongoing research and development, and the potential for achieving a supra-terawatt peak power in few-cycle pulses at a long-wave infrared wavelength with a laser system based on high-pressure, mixed-isotope $\mathrm{CO}_{2}$ amplifiers.
\end{abstract}

\section{Introduction}

Extending the wavelength range covered by high-peakpower laser systems into the mid-wave- and long-wave infrared (MWIR and LWIR respectively) promises many advantages for high-energy physics and material research [1]. Some examples of applications that can benefit from increasing the laser wavelength are the laser wakefield acceleration of electrons, the laser acceleration of ions from gas targets, and the generation of highenergy, supercontinuum, attosecond pulses. Despite the progress in the field of infrared solid-state sources and frequency conversion techniques [2], generation of short LWIR pulses at energy levels above a sub-millijoule is presently only possible using molecular gas lasers. We present here the state of the art in the multi-terawatt, picosecond LWIR laser technology by the example of a laser system based on high-pressure, mixed-isotope $\mathrm{CO}_{2}$ gas laser amplifiers operated at BNL and discuss the prospects for progressing towards few-cycle LWIR pulses of a supra-terawatt peak power.

\section{Background}

The carbon dioxide gas laser is historically one of the most used types of lasers due to its robustness, efficiency, and scalability. Pulsed atmospheric-pressure $\mathrm{CO}_{2}$ lasers transversely excited by an electric discharge (TEA $\mathrm{CO}_{2}$ lasers) can produce hundreds of joules of energy in long, typically a hundred of nanoseconds, pulses. The generation of short $(<1 \mathrm{~ns})$ pulses at such gas pressure is not possible due to a highly modulated rotationalvibrational structure of the gain spectrum shown in Fig. 1(a). A quasi-continuum gain spectrum shown in Fig. 1(b) can be achieved using an elevated gas pressure of $\sim 10$ bar for collisional broadening and admixing the ${ }^{18} \mathrm{O}$ containing isotopologues of $\mathrm{CO}_{2}$ for increasing the density of the spectral lines. The $\sim 1 \mathrm{THz}$ bandwidth of the strongest branch of this spectrum, $9 R$ centred at $9.2 \mu \mathrm{m}$ corresponds to the bandwidth of a transform-limited $500 \mathrm{fs}$ pulse. However, minimum pulse duration of an amplified pulse is restricted to $2 \mathrm{ps}$ due to the gain narrowing [3].

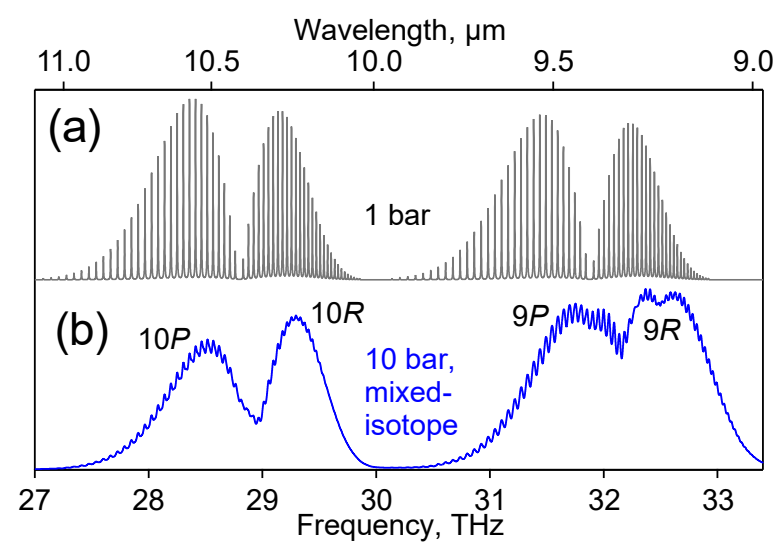

Fig. 1. Typical gain spectra of $\mathrm{CO}_{2}$ lasers: (a) conventional atmospheric-pressure laser; (b) high-pressure, mixed-isotope $\mathrm{C}^{16} \mathrm{O}_{2}-\mathrm{C}^{16} \mathrm{O}^{18} \mathrm{O}-\mathrm{C}^{18} \mathrm{O}_{2}$ laser.

\section{State of the art in a short-pulse $\mathrm{CO}_{2}$ laser technology}

Achieving a $15 \mathrm{TW}$ peak power in a $10.6 \mu \mathrm{m} 3 \mathrm{ps}$ (FWHM here and through the article) pulse using a $\mathrm{CO}_{2}$ laser system based on a $180 l$ active volume amplifier producing one shot in 30 minutes was reported by UCLA [4]. A relatively moderate, 2.5 bar gas pressure in this amplifier resulted in a train of pulses with 18 ps period with the highest peak power attained in the middle of the train.

An LWIR system with a higher, $1 \mathrm{~Hz}$, repetition rate, but of a lower, $1 \mathrm{TW}$, power based on much smaller, $\sim 1 l$ volume, 12 bar $\mathrm{CO}_{2}$ amplifiers is currently under development at the Naval Research Laboratory [5].

A presently operational single-pulse, multi-TW LWIR laser system at BNL's Accelerator Test Facility (ATF) features a sub-picosecond front end based on a solid-state

\footnotetext{
* Corresponding author: polyanskiy@bnl.gov
} 


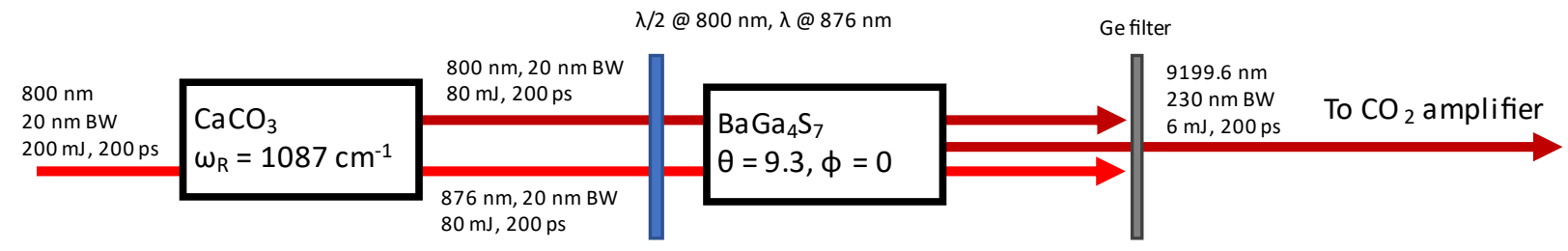

Fig. 2. Proposed seed generation scheme. A stretched $800 \mathrm{~nm}$ TiS laser pulse pumps a Calcite crystal to generate an $876 \mathrm{~nm}$ seed by stimulated Raman scattering. Polarization state of two NIR pulses is adjusted by a dual-wavelength waveplate. LWIR pulse is produced via type-I difference frequency generation in BGS and separated from the NIR pump by a Ge filter.

laser with an optical parametric amplifier that seeds a pulse into a series of two 8.5-10 bar, mixed-isotope $\mathrm{CO}_{2}$ amplifiers with $0.16 l$ and $8.5 l$ active volumes. A 5 TW peak power in a single 2 ps pulse has been demonstrated with this system [6] operated at up to one shot per 20 seconds repetition rate. This laser system is configured for a chirped-pulse amplification (CPA) regime to avoid detrimental wave front distortions at the output amplifier window. Much higher, up to $\mathrm{kHz}$, repetition rates might be possible from such laser systems [7].

\section{Quest for few-cycle, multi-TW LWIR pulses}

The near-term goal of the ongoing research at ATF is achieving a $10 \mathrm{TW}$ LWIR peak power in sub-picosecond pulses. In long term, few-cycle pulses (optical cycle duration at $9.2 \mu \mathrm{m}$ is $31 \mathrm{fs}$ ) with peak powers approaching $100 \mathrm{TW}$ is a challenging but not an unrealistic goal. Numerical modelling and initial proof-of-principle experiments show that the ultimate reduction of the pulse duration to $\sim 100 \mathrm{fs}$ is possible by combining two techniques:

1) Amplification of a $\sim 500 \mathrm{fs}$ pulse using a combination of the $9 P$ and $9 R$ branches of the gain spectrum [8], and

2) Post-compression of the pulse [9].

\subsection{Strong broad-band seed}

To achieve amplification of a broad-bandwidth pulse to multi-joule energies while avoiding substantial gain narrowing, total amplification in the $\mathrm{CO}_{2}$ amplifiers must be kept at a relatively low, $\sim 10^{3}$ level. Thus, the energy of the sub-picosecond seed pulse must be in the order of several millijoules. We are presently investigating a possibility for generating such a seed pulse using Raman conversion of a near-infrared pulse in a calcite $\left(\mathrm{CaCO}_{3}\right)$ crystal followed by difference frequency generation between the fundamental and Raman-shifted frequencies according to the scheme presented in Fig. 2.

Figure 3 shows the results of numerical modeling where a $300 \mathrm{fs}, 10 \mathrm{~mJ}$ pulse at $10.3 \mu \mathrm{m}$ central wavelength is amplified in the final ATF amplifier in CPA regime. The output pulse comprises a $500 \mathrm{fs}$ leading peak containing $75 \%$ of the energy followed by a small secondary peak at an 1 ps delay. The four-fold shortening of the pulse compared to the present configuration results in the corresponding increase in the peak power from $5 \mathrm{TW}$ to 20 TW.
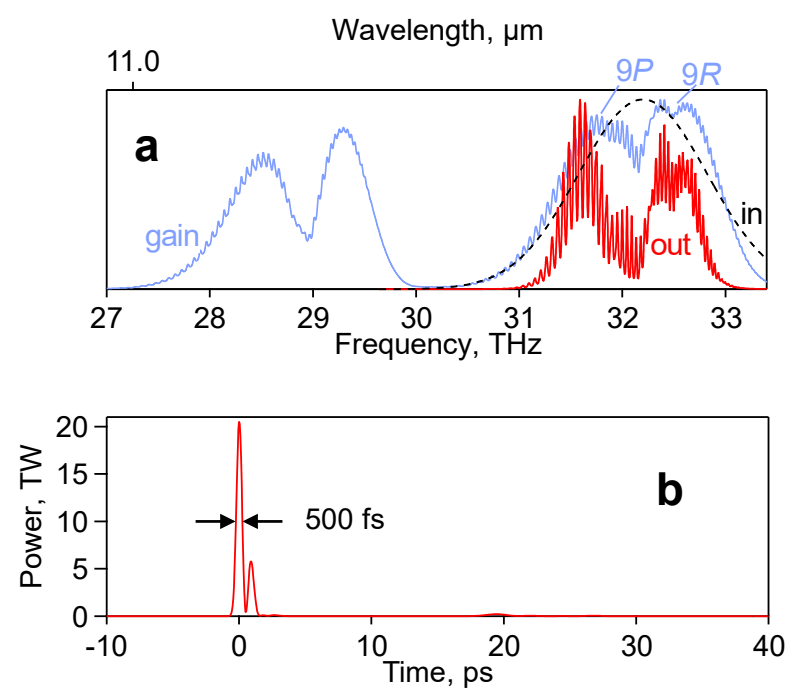

Fig. 3. Calculated spectrum (a, "out") and temporal pulse structure (b) of a $14 \mathrm{~J}$ pulse ( $20 \mathrm{~J}$ before compressor) with a $10 \mathrm{~mJ}, 300 \mathrm{fs}$ pulse seeded into the final amplifier of the ATF's laser system. The spectrum of the seed pulse and a typical gain spectrum of a high-pressure mixed-isotope $\mathrm{CO}_{2}$ amplifier are also shown in (a) as "in" and "gain," respectively.

\subsection{Post-compression}

The post-compression techniques for near-IR high-power laser pulses usually involve broadening of the pulse's spectrum via self-phase modulation (SPM) in a nonlinear material followed by the chirp compensation on a chirping mirror. Many relevant materials transparent to the LWIR exhibit a negative group velocity dispersion (GVD) allowing combining the SPM and the dispersive chirp compensation actions in one bulk optical slab [10].
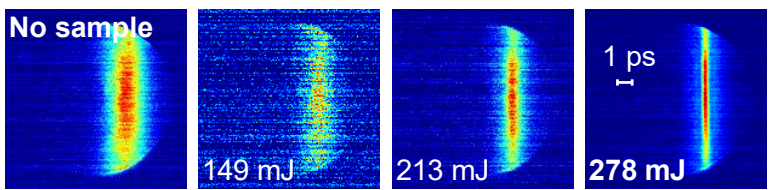

Fig. 4. Raw autocorrelation images of a pulse compressed in a $\mathrm{KCl}+\mathrm{BaF}_{2}$ (each $50 \mathrm{~mm}$ thick) post-compressor system at different energies.

We have recently proposed and experimentally demonstrated a modified version of this scheme where a 
combination of two materials, $\mathrm{KCl}$ and $\mathrm{BaF}_{2}$, with substantially different ratios of nonlinear refractive index to GVD is used to partially decouple the SPM and the dechirping actions and experimentally demonstrated compression of a 2 ps sub-TW pulse to $<500$ fs duration (see Fig. 4) [9].

For a $500 \mathrm{fs}$ pulse (Fig. 3b) at the input to a postcompressor, a few-cycle pulse can be expected at the output if the thickness of the elements is properly optimized. Calculations shown in Fig. 5 for input energy fluence of $500 \mathrm{~mJ} / \mathrm{cm}^{2}$ in a flat-top beam and $15 \mathrm{~mm}$ thick $\mathrm{KCl}$ and $\mathrm{BaF}_{2}$ plates indicate compression to $50 \mathrm{fs}$ (less than two optical cycles at $9.3 \mu \mathrm{m}$ ) with the peak power exceeding $100 \mathrm{TW}$.
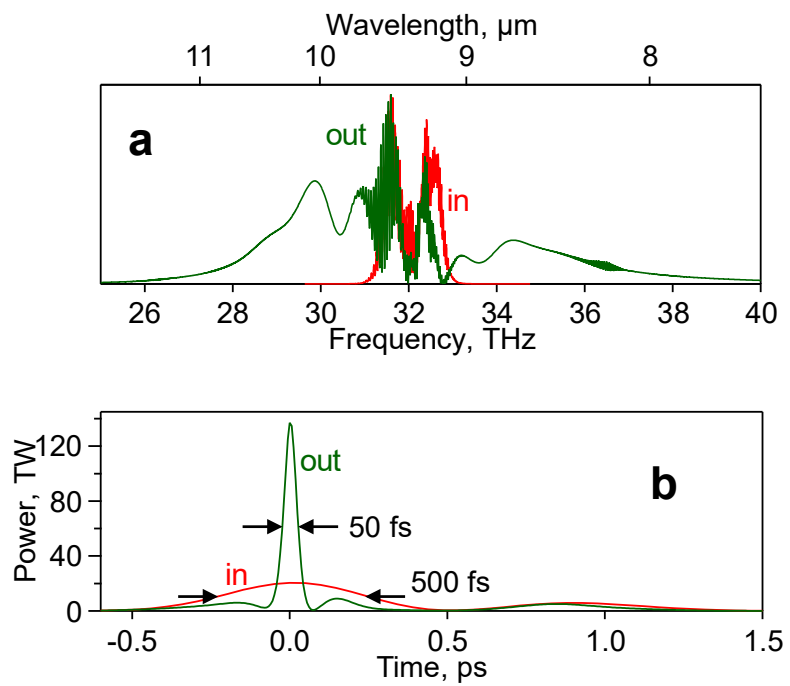

Fig. 5. Calculated spectrum (a) and temporal pulse structure (b) of a pulse before ("in") and after ("out") the post-compression in $15 \mathrm{~mm}$ thick $\mathrm{KCl}$ and $\mathrm{BaF}_{2}$ plates. Input pulse corresponds to the $500 \mathrm{fs}, 14 \mathrm{~J}$ output from the laser amplifier (Fig. 3 "out").

\section{Conclusions}

Both segments of the roadmap towards a few-cycle, supra-terawatt LWIR pulse, including an energetic subpicosecond seed generator and a post-compressor, require further research. Once conclusive results are obtained for each of these techniques, they will be combined in one laser system for achieving the ultimate performance.

Note that the prospects towards $>100$ TW ultra-fast LWIR laser systems described here are based on the parameters of a relatively modestly sized ATF $\mathrm{CO}_{2}$ laser. Up-scaling of the laser amplifier size and its output energy might allow to approach Petawatt power levels.

\section{Funding}

Department of Energy (DOE) Accelerator Stewardship Program grant, B\&R \#KA2601020. BNL Laboratory Directed Research and Development (LDRD) grant \#20$010 \mathrm{AD} / \mathrm{NPP}$.

\section{References}

1. D.J. Wilson, A.M. Summers, S. Zigo, B. Davis, S.-J. Robatjazi, J.A. Powell, D. Rolles, A. Rudenko, C.A. Trallero-Herrero, Sci. Rep. 9, 6002 (2019)

2. K.L. Vodopyanov, Laser based mid-infrared sources and applications (Wiley, 2020)

3. M.N. Polyanskiy, I.V. Pogorelsky, V. Yakimenko, Optica 2, 7717 (2011)

4. D. Haberberger, S. Tochitsky, C. Joshi, Opt. Express 18, 17865 (2011)

5. D.F. Gordon, V. Hasson, H. von Bergmann, Y.H. Chen, A. Schmitt-Sody, J.R. Penano, Proc. SPIE 9835, 98350Z-1 (2016)

6. M.N. Polyanskiy, I.V. Pogorelsky, M. Babzien, M.A. Palmer, OSA Continuum 3, 459 (2020)

7. I. V. Pogorelsky, M. Polyanskiy, T. Shaftan, Phys. Rev. Accel. Beams 23, 120702 (2020)

8. M.N. Polyanskiy, I.V. Pogorelsky, M. Babzien, R. Kupfer, N. Vafaei-Najafabadi, M.A. Palmer, Photonics 8, 101 (2021)

9. M.N. Polyanskiy, I.V. Pogorelsky, M. Babzien, R. Kupfer, K.L. Vodopyanov, M.A. Palmer, Postcompression of long-wave infrared 2 picosecond subterawatt pulses in bulk materials, submitted to Optics Express (2021)

10. B.G Bravy, V.M Gordienko, V.T. Platonenko, Laser Phys. Lett. 11, 065401 (2014) 